\title{
SAMPLE CONTINUITY AND MODELING OF STOCHASTIC PROCESSES FROM THE SPACES $D_{V, W}$
}

UDC 519.21

\author{
YU. V. KOZACHENKO AND O. M. MOKLYACHUK
}

\begin{abstract}
Random sequences and stochastic processes belonging to the spaces $D_{V, W}$ are studied in the paper. Conditions for the sample continuity of such processes are found. The convergence of series of random variables belonging to the spaces $D_{V, W}$ are considered. Models of stochastic processes belonging to the spaces $D_{V, W}$ are studied. Several examples of models are given.
\end{abstract}

\section{INTRODUCTION}

The spaces $D_{V, W}$ introduced in the paper [1] are defined as pre-Banach spaces generated by certain pre-metrics, namely by

$$
\|\xi\|=\sup _{x \geq 0} V(x) W^{(-1)}(\mathrm{P}\{|\xi|>x\}) .
$$

The basic properties of the spaces $D_{V, W}$, conditions for the convergence of series of random variables belonging to these spaces, and behavior of the supremum of stochastic processes in the spaces $D_{V, W}$ are considered in [1. In this paper, we continue studies of the spaces $D_{V, W}$ and stochastic processes belonging to these spaces.

In Section 1, we give basic definitions and results concerning the spaces $D_{V, W}$. Section 2 contains several other results on the random variables and stochastic processes belonging to the spaces $D_{V, W}$ that will be used in the later sections. The sample continuity of stochastic processes is studied in Section 3. Some results concerning the models of stochastic processes in $D_{V, W}$ are obtained in Section 4 the models approximate the initial processes with a given reliability and accuracy. Examples of models for some stochastic processes are discussed in Section 5.

\section{The SPACES $D_{V, W}$}

Let $\{\Omega, \mathcal{B}, \mathrm{P}\}$ be a standard probability space, $L_{0}(\Omega)$ the space of random variables defined on $\{\Omega, \mathcal{B}, \mathrm{P}\}$, and let $\mathcal{M} \subset L_{0}(\Omega)$ be some linear space.

Definition $1.1([2])$. A function $\Theta=(\Theta(\xi), \xi \in \mathcal{M})$ is called a pre-norm if, for all random variables $\xi \in \mathcal{M}$,

1. $\Theta(\xi) \in[0, \infty)$;

2. $\Theta(0)=0$;

3. $\Theta(-\xi)=\Theta(\xi)$.

2010 Mathematics Subject Classification. Primary 60G07.

Key words and phrases. Stochastic processes, modeling of stochastic processes, pre-norm, quasi-norm, pre-Banach spaces, quasi-Banach spaces, spaces $D_{V, W}$.

The first author is grateful to the Department of Mathematics and Statistics, La Trobe University, Melbourne, for support in the framework of a research grant "Stochastic Approximation in Finance and Signal Processing". 
Definition $1.2([2])$. If $\mathcal{M}$ is complete with respect to a pre-norm $\Theta$, then it is called a pre-Banach space.

Definition 1.3. A pre-Banach space $\mathcal{M}$ is called a pre- $K_{\sigma}$-space if

a1) $\max (\xi, \eta) \in \mathcal{M}$ and $\min (\xi, \eta) \in \mathcal{M}$ for all $\xi, \eta \in \mathcal{M}$ (this, in particular, means that $|\xi| \in \mathcal{M})$

a2) $|\xi| \in \mathcal{M}$ provided that $|\xi| \leq|\eta|$ and $\eta \in \mathcal{M}$.

Definition 1.4 (4]). Let every random variable $\xi \in \mathcal{M}$ correspond to a nonnegative number $\|\xi\|$ such that

1. $\|\xi\|=0 \Leftrightarrow \xi=0$ with probability one;

2. $\|\xi+\eta\| \leq\|\xi\|+\|\eta\|$;

3. if $|\lambda| \leq 1$, then $\|\lambda \xi\| \leq\|\xi\|$.

Then the functional $\|\cdot\|$ is called a quasi-norm.

Definition 1.5. If $\mathcal{M}$ is complete with respect to a quasi-norm $\|\cdot\|$, then $\mathcal{M}$ is called a quasi-Banach space.

Remark 1.1. Every quasi-norm is a pre-norm. If we assume that $\|\lambda \xi\|=|\lambda| \cdot\|\xi\|$ instead of condition 3 in Definition 1.4, then a quasi-norm is a usual norm.

Definition $1.6([3])$. A positive nondecreasing sequence $\mu(n), n \geq 1$, is called a majorizing characteristics of a pre-Banach $K_{\sigma}$-space $\mathcal{M}$ if

$$
\Theta\left(\max _{1 \leq k \leq n}\left|\xi_{k}\right|\right) \leq \mu(n) \max _{1 \leq k \leq n} \Theta\left(\xi_{k}\right)
$$

for all $\xi_{k} \in \mathcal{M}, k=1,2, \ldots, n$.

The notion of a characteristic is introduced in the papers [7] and [8] for Orlicz spaces, in [5] for $K_{\sigma}$-spaces, and in [6] for quasi-Banach $K_{\sigma}$-spaces.

Definition $1.7([3])$. Let $J=J(\lambda)$ be a nondecreasing function such that $J(\lambda) \geq 0$ and $J(\lambda) \rightarrow 0$ as $\lambda \rightarrow 0$. If a pre-norm $\Theta(\cdot)$ defined in $\mathcal{M}$ is such that

$$
\Theta(\lambda \xi) \leq J(|\lambda|) \Theta(\xi),
$$

then $\Theta$ is called a pre-norm subordinate to the function $J$.

Definition $1.8([2])$. A continuous even convex function $U=(U(x), x \in \mathbf{R})$ is called a $C$-function if $U(0)=0$ and $U(x)$ is increasing for $x>0$.

Now we define the space $D_{V, W}(\Omega)$.

Definition 1.9 ([1]). Let $W=\{W(x), x \in \mathbf{R}\}$ and $V=\{V(x), x \in \mathbf{R}\}$ be two functions such that $W(0)=0, W(x)>0$, and $V(x)>0$ for $x \neq 0$. Moreover, we assume that both functions are even, increasing, and continuous for $x>0$. Let there exist a constant $C>0$ and a continuous function $Z=\{Z(x), x>0\}$ such that

$$
\begin{gathered}
W^{(-1)}(x+y) \leq C\left(W^{(-1)}(x)+W^{(-1)}(y)\right), \\
V(a x) \leq Z(a) V(x)
\end{gathered}
$$

for $x>0$ and for all constants $a>0$, and

$$
0<Z(x)<\infty
$$

for $|x|<\infty$. We say that a random variable $\xi$ belongs to the space $D_{V, W}(\Omega)$ if

$$
\sup _{x \geq 0} V(x) W^{(-1)}(\mathrm{P}\{|\xi|>x\})<\infty .
$$


Examples of functions $W$ and $V$ with the above properties are $W(x)=|x|^{a}$ or $W(x)=$ $\exp \left\{|x|^{a}\right\}-1, a>0$, and $V(x)=|x|^{b}, b>0$.

Theorem 1.1 ([1]). The space $D_{V, W}(\Omega)$ is a pre- $K_{\sigma}$-space with respect to the following pre-norm:

$$
\|\xi\|_{V, W}=\left(\sup _{x>0} V(x) W^{-1}(\mathrm{P}\{|\xi|>x\})\right)^{1 / 2} .
$$

If $\left\|\xi_{n}-\xi_{m}\right\|_{V, W} \rightarrow 0$ as $n, m \rightarrow \infty$ and $\sup _{n}\left\|\xi_{n}\right\|_{V, W}<\infty$, then there exists a random variable $\xi \in D_{V, W}(\Omega)$ such that $\left\|\xi_{n}-\xi\right\|_{V, W} \rightarrow 0$ as $n \rightarrow \infty$. Moreover, the pre-norm $\|\cdot\|_{V, W}$ is subordinate to the function $J(\lambda)=(Z(\lambda))^{1 / 2}$.

Let $W(x)$ be an Orlicz $C$-function and let $V(x)$ be the inverse to an Orlicz $C$-function. Then the functional $\|\cdot\|$ is a quasi-norm and the space is complete with respect to this quasi-norm.

Finally,

$$
\mathrm{P}\{|\xi|>x\} \leq W\left(\frac{\|\xi\|_{V, W}^{2}}{V(x)}\right)
$$

for all $x>0$.

Theorem 1.2 ([1]). The sequence

$$
\mu(n)=\sup _{0<t<1 / n}\left(\frac{W^{(-1)}(t n)}{W^{(-1)}(t)}\right)^{1 / 2}
$$

is a majorizing characteristic of the space $D_{V, W}(\Omega)$.

2. Properties of SERIES of RANDOM VARIABLES AND STOCHASTIC PROCESSES BELONGING TO THE SPACES $D_{V, W}$

Theorem 2.1 ([1]). Let $\xi_{k}$ be random variables belonging to $D_{V, W}(\Omega),\|\cdot\|$ be a prenorm such that $\left\|\xi_{k}\right\|>0, f(x)=x V(W(x)), x>0$, and let $f^{(-1)}(x)$ be the inverse to the function $f(x)$. The series

$$
\sum_{k=1}^{\infty} \xi_{k}
$$

converges in probability if the series

$$
\sum_{k=1}^{\infty} \alpha_{k}^{*}
$$

converges, where

Moreover, if

$$
\alpha_{k}^{*}=V^{(-1)}\left(\frac{\left\|\xi_{k}\right\|^{2}}{f^{(-1)}\left(\left\|\xi_{k}\right\|^{2}\right)}\right)
$$

$$
x \geq \mu=\sum_{k=1}^{\infty} V^{(-1)}\left(\frac{\left\|\xi_{k}\right\|^{2}}{f^{(-1)}\left(\left\|\xi_{k}\right\|^{2}\right)}\right),
$$

then

$$
\mathrm{P}\left\{\left|\sum_{k=1}^{\infty} \xi_{k}\right| \geq x\right\} \leq \sum_{k=1}^{\infty} W\left(\frac{\left\|\xi_{k}\right\|^{2}}{V\left(\frac{\alpha_{k}^{*} x}{\mu}\right)}\right),
$$

where the series on the right hand side of (5) converges for $x \geq \mu$. 
Remark 2.1. The function $x / f^{(-1)}(x)$ increases, since the function $f(x) / x=V(W(x))$ increases.

Theorem 2.2 ([1]). Let $W(x)=|x|^{a}, a>0$, and let $V(x)=|x|^{b}, b>0$. Then series (3) converges in probability if the series

$$
\mu=\sum_{k=1}^{\infty}\left\|\xi_{k}\right\|^{2 a /(a b+1)}
$$

converges. Moreover,

$$
\mathrm{P}\left\{\left|\sum_{k=1}^{\infty} \xi_{k}\right|>x\right\} \leq \frac{1}{x^{a b}}\left(\sum_{k=1}^{\infty}\left\|\xi_{k}\right\|^{2 a /(a b+1)}\right)^{a b+1}
$$

for $x \geq \mu$, that is, $\sum_{k=1}^{\infty} \xi_{k}$ belongs to the space $D_{V, W}$, and

$$
\left\|\sum_{k=1}^{\infty} \xi_{k}\right\| \leq\left(\sum_{k=1}^{\infty}\left\|\xi_{k}\right\|^{2 a /(a b+1)}\right)^{(a b+1) /(2 a)} .
$$

Definition 2.1 ([1]). We say that a stochastic process $X(t)=\{X(t), t \in T\}$ belongs to the space $D_{V, W}$ if $X(t) \in D_{V, W}$ for all $t$.

The processes represented in the form

$$
\xi(t)=\sum_{k=1}^{\infty} \xi_{k} \phi_{k}(t), \quad t \in T
$$

are examples of stochastic processes belonging to the space $D_{V, W}$ if $\xi_{k} \in D_{V, W}$ and if the latter series converges in the space $D_{V, W}$.

Conditions for the convergence of series in (6) are presented in [1].

Definition $2.2([2])$. A function $\rho(t, s), t, s \in T$, is called a quasi-metric if $\rho(t, s) \in$ $[0, \infty), \rho(t, t)=0$, and $\rho(t, s)=\rho(s, t)$.

Let $X=\{X(t), t \in T\}$ be a stochastic process belonging to the space $D_{V, W}$. Then $\rho_{X}(t, s)=\|X(s)-X(t)\|$ is called the pre-metric generated by the process $X$.

Let a process $X$ be such that

(A1) $\sup _{t \in T}\|X(t)\|<\infty$;

(A2) the space $\left(T, \rho_{X}\right)$ is separable and $X$ is a separable process in $\left(T, \rho_{X}\right)$.

Put $\varepsilon_{0}=\sup _{t, s \in T} \rho_{X}(t, s)$. Condition (A1) implies that $\varepsilon_{0}<\infty$. Let $\theta \in(0,1)$, $\varepsilon_{k}=\varepsilon_{0} \theta^{k}$, and let $N(\varepsilon)$ be the metric capacity of the space $(T, \rho)$, that is, $N(\varepsilon)$ is the minimum number of closed balls covering $(T, \rho)$.

The following result contains conditions for $\sup _{t \in T} X(t)<\infty$ with probability one as well as estimates for the distribution of this supremum.

Theorem 2.3 ([1]). Let a stochastic process $X$ satisfy conditions (A1) and (A2). If the series

$$
\sum_{n=1}^{\infty} V^{(-1)}\left(\frac{\mu\left(N\left(\varepsilon_{n}\right)\right)^{2} \varepsilon_{n-1}^{2}}{f^{(-1)}\left(\mu\left(N\left(\varepsilon_{n}\right)\right)^{2} \varepsilon_{n-1}^{2}\right)}\right)
$$

converges, where $f$ is defined in Theorem 2.1, then

$$
\mathrm{P}\left\{\sup _{t \in T}|X(t)| \geq x\right\} \leq W\left(\frac{\inf _{t \in T}\|X(t)\|^{2}}{V\left(\psi_{0} x\right)}\right)+\sum_{k=1}^{\infty} W\left(\frac{\mu\left(N\left(\varepsilon_{k}\right)\right)^{2} \varepsilon_{k-1}^{2}}{V\left(\psi_{k} x\right)}\right),
$$


where

$$
\begin{gathered}
\psi_{0}=\frac{1}{\Psi} V^{(-1)}\left(\frac{\inf _{t \in T}\|X(t)\|^{2}}{f^{(-1)}\left(\inf _{t \in T}\|X(t)\|^{2}\right)}\right), \quad \psi_{k}=\frac{1}{\Psi} V^{(-1)}\left(\frac{\mu\left(N\left(\varepsilon_{k}\right)\right)^{2} \varepsilon_{k-1}^{2}}{f^{(-1)}\left(\mu\left(N\left(\varepsilon_{k}\right)\right)^{2} \varepsilon_{k-1}^{2}\right)}\right), \\
\Psi=\sum_{k=0}^{\infty} \psi_{k}, \quad x>\Psi .
\end{gathered}
$$

Theorem 2.4 ([1]). Let a stochastic process $X=\{X(t), t \in T\}$ be such that $X \in$ $D_{V, W}$ and let $W(x)=|x|^{a}, a>0$, and $V(x)=|x|^{b}, b>0$. Assume that $X$ satisfies conditions (A1) and (A2).

If

$$
\int_{0}^{\Delta_{0} p}\left(N\left(u^{(a b+1) /(2 a)}\right)\right)^{1 /(a b+1)} d u<\infty,
$$

where $p=\theta^{2 a /(a b+1)}, 0<\theta<1, \Delta_{0}=\varepsilon_{0}^{2 a /(a b+1)}$, and $\varepsilon_{0}=\sup _{t, s \in T} \rho_{X}(t, s)$, then

$$
\sup _{t \in T}|X(t)| \in D_{V, W}
$$

and, moreover,

$$
\begin{aligned}
& \mathrm{P}\left\{\sup _{t \in T}|X(t)| \geq x\right\} \\
& \quad \leq \frac{1}{x^{a b}}\left(\inf _{t \in T}\|X(t)\|^{2 a /(a b+1)}+\frac{1}{p(1-p)} \int_{0}^{\Delta_{0} p}\left(N\left(u^{(a b+1) /(2 a)}\right)\right)^{1 /(a b+1)} d u\right) .
\end{aligned}
$$

3. The continuity of stochastic processes belonging to the spaces $D_{V, W}$

Let $X$ be a stochastic process belonging to the space $D_{V, W}$ such that

$$
\sup _{t \in T}\|X(t)\|<\infty
$$

Let $\rho_{X}(t, s)=\|X(t)-X(s)\|$ be the quasi-metric generated by the process $X$. Also let $\left(T, \rho_{X}\right)$ be a separable space and $X$ be a separable process in $\left(T, \rho_{X}\right)$.

Let $\theta \in(0,1)$ and $\varepsilon_{k}=\varepsilon_{0} \theta^{k}, k \geq 1$, where

$$
\varepsilon_{0}=\sup _{t, s \in T}\|X(t)-X(s)\| .
$$

By $V_{\varepsilon_{k}}$, we denote the set of centers of closed balls of radius $\varepsilon_{k}$ that form a minimal covering of the space $(T, \rho)$. The cardinality of the set $V_{\varepsilon_{k}}$ is equal to $N\left(\varepsilon_{k}\right)$. Let $t, s \in T$ be some points such that $\rho(t, s)<\varepsilon$ for $0<\varepsilon<\varepsilon_{0}$.

Now we find $k$ such that $\varepsilon_{k}<\varepsilon<\varepsilon_{k-1}$. Then

$$
V_{k}=\bigcup_{j=k}^{\infty} V_{\varepsilon_{j}}
$$

is the set of separability of the process $X(t)$, since $X(t)$ is continuous in probability.

By $S_{n}$, we denote the minimal $\varepsilon_{n}$-net of the set $T$ with respect to the pseudo-metric $\rho_{x}$. Put $S=\bigcup_{n=0}^{\infty} S_{n}$.

Definition $3.1([2])$. A family of mappings $\alpha_{k}(t), k=0,1, \ldots$, is called an $\alpha$-procedure if every point of $S$ corresponds to a unique point $\alpha_{k}$ of $S_{k}$ such that $\rho\left(t, \alpha_{k}(t)\right) \leq \varepsilon_{k}$. 
Theorem 3.1. Assume that a stochastic process $X$ satisfies all the above conditions. If the following two series

$$
\sum_{l=k}^{\infty} V^{(-1)}\left(\frac{\mu^{2}\left(N^{2}\left(\varepsilon_{l}\right)\right) \varepsilon_{l-1}^{2}}{\left.f^{(-1)} \mu^{2}\left(N^{2}\left(\varepsilon_{l}\right)\right) \varepsilon_{l-1}^{2}\right)}\right)
$$

and

$$
\sum_{l=k}^{\infty} V^{(-1)}\left(\frac{\varepsilon_{l-1}^{2}}{f^{(-1)}\left(\varepsilon_{l-1}^{2}\right)}\right)
$$

converge and $x \geq \Psi$, where

$$
\Psi=V^{(-1)}\left(\frac{\mu^{2}\left(N^{2}\left(\varepsilon_{k}\right)\right) \hat{\varepsilon}^{2}}{f^{(-1)}\left(\mu^{2}\left(N^{2}\left(\varepsilon_{k}\right)\right) \hat{\varepsilon}^{2}\right)}\right)+\sum_{l=k}^{\infty} V^{(-1)}\left(\frac{\mu^{2}\left(N^{2}\left(\varepsilon_{l}\right)\right) \varepsilon_{l-1}^{2}}{f^{(-1)}\left(\mu^{2}\left(N^{2}\left(\varepsilon_{l}\right)\right) \varepsilon_{l-1}^{2}\right)}\right),
$$

then

$$
\begin{aligned}
& \mathrm{P}\left\{\sup _{\rho(t, s)<\varepsilon}|X(t)-X(s)| \geq x\right\} \\
& \quad \leq W\left(\frac{\mu^{2}\left(N^{2}\left(\varepsilon_{k}\right)\right) \hat{\varepsilon}_{k}^{2}}{V\left(\psi_{0} x\right)}\right)+\sum_{l=k}^{\infty} W\left(\frac{\mu^{2}\left(N^{2}\left(\varepsilon_{l}\right)\right) \varepsilon_{l-1}^{2}}{V\left(\psi_{l} x\right)}\right),
\end{aligned}
$$

where

$$
\begin{aligned}
& \psi_{0}=\frac{1}{\Psi} V^{(-1)}\left(\frac{\mu^{2}\left(N^{2}\left(\varepsilon_{k}\right)\right) \hat{\varepsilon}^{2}}{f^{(-1)}\left(\mu^{2}\left(N^{2}\left(\varepsilon_{k}\right)\right) \hat{\varepsilon}^{2}\right)}\right), \\
& \psi_{l}=\frac{1}{\Psi} V^{(-1)}\left(\frac{\mu^{2}\left(N^{2}\left(\varepsilon_{l}\right)\right) \varepsilon_{l-1}^{2}}{f^{(-1)}\left(\mu^{2}\left(N^{2}\left(\varepsilon_{l}\right)\right) \varepsilon_{l-1}^{2}\right.}\right),
\end{aligned}
$$

and

$$
\hat{\varepsilon}=\varepsilon \frac{5-3 \theta}{1-\theta} .
$$

Moreover $X(t)$ is a sample-continuous stochastic process in the space $(T, \rho)$.

Proof. Let $m>k$ be an arbitrary number. Consider the points

$$
t_{m}=\alpha_{m}(t), \quad t_{m-1}=\alpha_{m-1}\left(t_{m}\right), \quad \ldots, \quad t_{k}=\alpha_{k}\left(t_{k+1}\right)
$$

and

$$
s_{m}=\alpha_{m}(t), \quad s_{m-1}=\alpha_{m-1}\left(s_{m}\right), \quad \ldots, \quad s_{k}=\alpha_{k}\left(s_{k+1}\right),
$$

where $\alpha_{k}(t)$ is an $\alpha$-procedure. Then

$$
\begin{aligned}
X(t)- & X(s) \\
= & \left(X(t)-X\left(\alpha_{m}(t)\right)\right)+\left(X(s)-X\left(\alpha_{m}(s)\right)\right) \\
& +\sum_{l=k}^{m-1}\left(X\left(t_{l+1}\right)-X\left(t_{l}\right)\right)+\sum_{l=k}^{m-1}\left(X\left(s_{l+1}\right)-X\left(s_{l}\right)\right)+\left(X\left(t_{k}\right)-X\left(s_{k}\right)\right) .
\end{aligned}
$$


This implies that

$$
\begin{aligned}
\mathrm{P}\{\mid X( & \left.\left.t_{k}\right)-X\left(s_{k}\right) \mid \geq x\right\} \\
\leq & \mathrm{P}\left\{\left|X(t)-X\left(\alpha_{m}(t)\right)\right|>x \psi_{1}^{\prime}\right\}+\mathrm{P}\left\{\left|X(s)-X\left(\alpha_{m}(s)\right)\right|>x \psi_{1}^{\prime \prime}\right\} \\
& +\sum_{l=k}^{m-1} \mathrm{P}\left\{\left|X\left(t_{l+1}\right)-X\left(t_{l}\right)\right|>x \psi_{l}^{\prime}\right\}+\sum_{l=k}^{m-1} \mathrm{P}\left\{\left|X\left(s_{l+1}\right)-X\left(s_{l}\right)\right|>x \psi_{l}^{\prime \prime}\right\} \\
& +\mathrm{P}\left\{|X(t)-X(s)|>x \psi_{0}\right\} \\
\leq & W\left(\frac{\left\|X(t)-X\left(\alpha_{m}(t)\right)\right\|^{2}}{V\left(x \psi_{1}^{\prime}\right)}\right)+W\left(\frac{\left\|X(s)-X\left(\alpha_{m}(s)\right)\right\|^{2}}{V\left(x \psi_{1}^{\prime \prime}\right)}\right) \\
& +\sum_{l=k}^{m-1} W\left(\frac{\left\|X\left(t_{l}\right)-X\left(\alpha_{l-1}\left(t_{l}\right)\right)\right\|^{2}}{V\left(x \psi_{l}^{\prime}\right)}\right) \\
& +\sum_{l=k}^{m-1} W\left(\frac{\left\|X\left(s_{l}\right)-X\left(\alpha_{l-1}\left(s_{l}\right)\right)\right\|^{2}}{V\left(x \psi_{l}^{\prime \prime}\right)}\right) \\
& +W\left(\frac{\|X(t)-X(s)\|^{2}}{V\left(x \psi_{0}\right)}\right) \\
\leq & 2 W\left(\frac{\varepsilon_{m-1}^{2}}{V\left(x \psi_{1}\right)}\right)+2 \sum_{l=k}^{m-1} W\left(\frac{\varepsilon_{l-1}^{2}}{V\left(x \psi_{l}\right)}\right)+W\left(\frac{\varepsilon_{0}^{2}}{V\left(x \psi_{0}\right)}\right)
\end{aligned}
$$

since $\left\|X(t)-X\left(\alpha_{n-1}(t)\right)\right\| \leq \varepsilon_{n-1}$.

Then equality (9) implies that

$$
\begin{aligned}
\left|X\left(t_{k}\right)-X\left(s_{k}\right)\right| \leq & \left|X(t)-X\left(\alpha_{m}(t)\right)\right|+\left|X(s)-X\left(\alpha_{m}(s)\right)\right| \\
& +\sum_{l=k}^{m-1}\left|X\left(t_{l+1}\right)-X\left(t_{l}\right)\right|+\sum_{l=k}^{m-1}\left|X\left(s_{l+1}\right)-X\left(s_{l}\right)\right|+|X(t)-X(s)| \\
\leq & 2 \sum_{l=k}^{m-1} \max _{u \in V_{\varepsilon_{l}}}\left|X(u)-X\left(\alpha_{l}(u)\right)\right|+\left|X(t)-X\left(\alpha_{k}(t)\right)\right| \\
& +\left|X(s)-X\left(\alpha_{k}(s)\right)\right|+|X(t)-X(s)| \\
\leq & 2 \sum_{l=k}^{m-1} \varepsilon_{l}+2 \varepsilon_{k}+\varepsilon \leq \hat{\varepsilon},
\end{aligned}
$$

where

$$
\hat{\varepsilon}=\varepsilon \frac{5-3 \theta}{1-\theta} .
$$

Passing to the limit in (9) as $m \rightarrow \infty$ we get

$$
\begin{aligned}
\sup _{\rho(t, s) \leq \varepsilon}|X(t)-X(s)| & =\sup _{|t-s| \leq \varepsilon, t, s, \in V}|X(t)-X(s)| \\
& \leq \max _{v, w \in V_{k}}|X(v)-X(w)|+2 \sum_{l=k}^{\infty} \max _{u \in V_{l+1}}\left|X(u)-X\left(\alpha_{l}(u)\right)\right|
\end{aligned}
$$

provided inequality (10) holds. 
After some transformations we obtain

$$
\begin{aligned}
\mathrm{P}\left\{\sup _{\rho(t, s) \leq \varepsilon}|X(t)-X(s)| \geq x\right\} \leq & \mathrm{P}\left\{\max _{v, w \in V_{k}}|X(v)-X(w)| \geq \psi_{0} x\right\} \\
& +\sum_{l=k}^{\infty} \mathrm{P}\left\{\max _{u \in V_{l+1}}\left|X(u)-X\left(\alpha_{l}(u)\right)\right| \geq \psi_{l} x\right\} .
\end{aligned}
$$

Reasoning similarly to the proof of Theorem 2.3 (see [1])) we get

$$
\begin{aligned}
& \mathrm{P}\left\{\sup _{\rho(t, s) \leq \varepsilon}|X(t)-X(s)| \leq x\right\} \\
& \quad \leq W\left(\frac{\mu^{2}\left(N^{2}\left(\varepsilon_{k}\right)\right) \hat{\varepsilon}_{k}^{2}}{V\left(\psi_{0} x\right)}\right)+\sum_{l=k}^{\infty} W\left(\frac{\mu^{2}\left(N^{2}\left(\varepsilon_{l}\right)\right) \varepsilon_{l-1}^{2}}{V\left(\psi_{l} x\right)}\right),
\end{aligned}
$$

where

$$
\begin{gathered}
\psi_{0}=\frac{1}{\Psi} V^{(-1)}\left(\frac{\mu^{2}\left(N^{2}\left(\varepsilon_{k}\right)\right) \hat{\varepsilon}^{2}}{f^{(-1)}\left(\mu^{2}\left(N^{2}\left(\varepsilon_{k}\right)\right) \hat{\varepsilon}^{2}\right)}\right), \\
\psi_{l}=\frac{1}{\Psi} V^{(-1)}\left(\frac{\mu^{2}\left(N^{2}\left(\varepsilon_{l}\right)\right) \varepsilon_{l-1}^{2}}{f^{(-1)}\left(\mu^{2}\left(N^{2}\left(\varepsilon_{l}\right)\right) \varepsilon_{l-1}^{2}\right.}\right), \\
\Psi=V^{(-1)}\left(\frac{\mu^{2}\left(N^{2}\left(\varepsilon_{k}\right)\right) \hat{\varepsilon}^{2}}{f^{(-1)}\left(\mu^{2}\left(N^{2}\left(\varepsilon_{k}\right)\right) \hat{\varepsilon}^{2}\right)}\right)+\sum_{l=k}^{\infty} V^{(-1)}\left(\frac{\mu^{2}\left(N^{2}\left(\varepsilon_{l}\right)\right) \varepsilon_{l-1}^{2}}{f^{(-1)}\left(\mu^{2}\left(N^{2}\left(\varepsilon_{l}\right)\right) \varepsilon_{l-1}^{2}\right.}\right) .
\end{gathered}
$$

Since $W(x)$ increases for $x>0$,

$$
W\left(\frac{\mu^{2}\left(N^{2}\left(\varepsilon_{k}\right)\right) \hat{\varepsilon}^{2}}{V\left(\psi_{0} x\right)}\right) \rightarrow 0
$$

if $x$ is fixed. Since the series

$$
\sum_{l=k}^{\infty} W\left(\frac{\mu^{2}\left(N^{2}\left(\varepsilon_{l}\right)\right) \varepsilon_{l-1}^{2}}{V\left(\psi_{l} x\right)}\right)
$$

converges, we pass to the limit as $k \rightarrow \infty$ and obtain

$$
W\left(\frac{\mu^{2}\left(N^{2}\left(\varepsilon_{k}\right)\right) \hat{\varepsilon}^{2}}{V\left(\psi_{0} x\right)}\right)+\sum_{l=k}^{\infty} W\left(\frac{\mu^{2}\left(N^{2}\left(\varepsilon_{l}\right)\right) \varepsilon_{l-1}^{2}}{V\left(\psi_{l} x\right)}\right) \rightarrow 0
$$

whence

$$
\mathrm{P}\left\{\sup _{\rho(t, s)<\varepsilon}|X(t)-X(s)| \geq x\right\} \rightarrow 0
$$

as $k \rightarrow \infty$.

This implies that the process is sample-continuous in $(T, \rho)$.

Theorem 3.2. Let $W(x)=x^{a}$, $a>1$, and $V(x)=x^{b}, 0<b<1$. If

$$
\int_{0}^{\Delta_{0} p^{k+1}} N\left(u^{(a b+1) /(2 a)}\right)^{2 /(a b+1)} d u<\infty,
$$


then

$$
\begin{aligned}
& \mathrm{P}\left\{\sup _{\rho(t, s)<\varepsilon}|X(s)-X(t)| \geq x\right\} \\
& \leq \frac{1}{x^{a b} p(1-p)}\left(\hat{C} \int_{\Delta_{0} p^{k+1}}^{\Delta_{0} p^{k}} N\left(u^{(a b+1) /(2 a)}\right)^{2 /(a b+1)} d u\right. \\
&\left.+2 \int_{0}^{\Delta_{0} p^{k+1}} N\left(u^{(a b+1) /(2 a)}\right)^{2 /(a b+1)} d u\right),
\end{aligned}
$$

where

$$
\hat{C}=\left(\frac{5-3 \theta}{1-\theta}\right)^{2 /(a b+1)} .
$$

Moreover, $X(t)$ is a sample-continuous stochastic process in $(T, \rho)$.

Proof. Reasoning as in the proof of Theorem 2.3 (see [1]) we get

$$
\begin{aligned}
& \mathrm{P}\left\{\sup _{\rho(t, s)<\varepsilon}|X(s)-X(t)| \geq x\right\} \\
& \leq \frac{1}{x^{a b} p(1-p)}\left(\left(\mu\left(N^{2}\left(\varepsilon_{k}\right)\right) \hat{\varepsilon}\right)^{2 a /(a b+1)}+2 \sum_{l=k+1}^{\infty}\left(\mu\left(N^{2}\left(\varepsilon_{l}\right)\right) \varepsilon_{l-1}\right)^{2 a /(a b+1)}\right) \\
& \leq \frac{1}{x^{a b} p(1-p)}\left(\int_{\Delta_{0} p^{k+1}}^{\Delta_{0} p^{k}} \mu\left(N^{2}\left(u^{(a b+1) /(2 a)}\right)\left(\frac{5-3 \theta}{1-\theta}\right)^{2}\right)^{2 a /(a b+1)} d u\right. \\
& \left.\quad+2 \int_{0}^{\Delta_{0} p^{k+1}} \mu\left(N^{2}\left(u^{(a b+1) /(2 a)}\right)\right)^{2 a /(a b+1)} d u\right)
\end{aligned}
$$

where the numbers $\Delta_{0}$ and $p$ are defined in the proof of Theorem 2.3 in [1].

Theorem 1.2 implies that

$$
\mu(n)=\sup _{0<t<1 / n}\left(\frac{W^{(-1)}(t n)}{W^{(-1)}(t)}\right)^{1 / 2}=n^{1 / 2 a} .
$$

Then

$$
\begin{gathered}
\mathrm{P}\left\{\sup _{\rho(t, s)<\varepsilon}|X(s)-X(t)| \geq x\right\} \\
\leq \frac{1}{x^{a b} p(1-p)}\left(\hat{C} \int_{\Delta_{0} p^{k+1}}^{\Delta_{0} p^{k}}\left(N\left(u^{(a b+1) /(2 a)}\right)\right)^{2 /(a b+1)} d u\right. \\
\left.\quad+2 \int_{0}^{\Delta_{0} p^{k+1}}\left(N^{2}\left(u^{(a b+1) /(2 a)}\right)\right)^{2 /(a b+1)} d u\right) \\
\leq \frac{C}{x^{a b} p(1-p)} \int_{0}^{\Delta_{0} p^{k+1}} N\left(u^{(a b+1) /(2 a)}\right)^{2 /(a b+1)} d u .
\end{gathered}
$$

Theorem 3.3. Let $X=\{X(t), t \in[0, T]\}$ be a stochastic process such that $X \in D_{V, W}$. Assume that $X$ is a separable process in $[0, T]$. Let $W(x)=|x|^{a}, a>0$, and $V(x)=|x|^{b}$, $b>0$. If

$$
\sup _{|t-s| \leq h}\|X(t)-X(s)\| \leq D h^{\zeta}
$$


for some $D>0$ and $\zeta>1 / a$, then

$$
\sup _{t \in[0, T]}|X(t)| \in D_{V, W}
$$

and

$$
\begin{aligned}
\mathrm{P}\left\{\sup _{\rho(t, s) \leq \varepsilon}|X(s)-X(t)| \geq x\right\} & \\
\leq & \frac{1}{x^{a b} p(1-p)}\left(\hat{C} \int_{\Delta_{0} p^{k+1}}^{\Delta_{0} p^{k}}\left(\frac{D T}{2 u^{(a b+1) /(2 a \zeta)}}+1\right)^{2 /(a b+1)} d u\right. \\
& \left.+2 \int_{0}^{\Delta_{0} p^{k+1}}\left(\frac{D T}{2 u^{(a b+1) /(2 a \zeta)}}+1\right)^{2 /(a b+1)} d u\right)
\end{aligned}
$$

for all $x>0$, where $\hat{C}$ is defined in (12).

Moreover, $X(t)$ is a sample-continuous stochastic process in $(T, \rho)$.

Proof. The assumptions of the theorem imply that

$$
N(\varepsilon) \leq \frac{D T}{2 \varepsilon^{1 / \zeta}}+1
$$

Inequality (11) can be used to obtain the following estimates:

$$
\begin{aligned}
& \mathrm{P}\left\{\sup _{\rho(t, s)<\varepsilon}|X(s)-X(t)| \geq x\right\} \\
& \leq \frac{1}{x^{a b} p(1-p)}\left(\hat{C} \int_{\Delta_{0} p^{k+1}}^{\Delta_{0} p^{k}}\left(N\left(u^{(a b+1) /(2 a)}\right)\right)^{2 /(a b+1)} d u\right. \\
& \left.\quad+2 \int_{0}^{\Delta_{0} p^{k+1}}\left(N\left(u^{(a b+1) /(2 a)}\right)\right)^{2 /(a b+1)} d u\right) \\
& \leq \frac{1}{x^{a b} p(1-p)}\left(\hat{C} \int_{\Delta_{0} p^{k+1}}^{\Delta_{0} p^{k}}\left(\frac{D T}{\left.2 u^{(a b+1) /(2 a \zeta)}+1\right)^{2 /(a b+1)} d u} d u\right) .\right.
\end{aligned}
$$

The two latter integrals converge if so does the integral

$$
\int_{0}^{\Delta_{0} p^{k+1}} \frac{1}{u^{1 /(a \zeta)}} d u
$$

which is the case if $\zeta>1 / a$.

The integrals

$$
\int_{0}^{\Delta_{0} p^{k+1}}\left(\frac{D T}{2 u^{(a b+1) /(2 a \zeta)}}+1\right)^{2 /(a b+1)} d u
$$

and

$$
\int_{\Delta_{0} p^{k+1}}^{\Delta_{0} p^{k}}\left(\frac{D T}{2 u^{(a b+1) /(2 a \zeta)}}+1\right)^{2 /(a b+1)} d u
$$

can be estimated via the hypergeometric function. 
4. Models of stochastic processes belonging to the spaces $D_{V, W}$

Consider a stochastic process $X$ represented in the following form:

$$
X(t)=\sum_{k=1}^{\infty} \xi_{k} \phi_{k}(t)
$$

for $t \in[0, T]$. We also consider another process $X_{N}$ given by

$$
X_{N}(t)=\sum_{k=1}^{N} \xi_{k} \phi_{k}(t) \text {. }
$$

Then $X_{N}(t)$ is called a model of the process $X$.

Put

$$
\tilde{X}_{N}(t):=\sum_{k=N+1}^{\infty} \xi_{k} \phi_{k}(t)=X(t)-X_{N}(t) .
$$

Theorem 4.1. Let $X=\{X(t), t \in[0, T]\}$ be a stochastic process represented in the form of (13) and such that $\xi_{k} \in D_{V, W}$. Let $W(x)=|x|^{a}, a>1$, and $V(x)=|x|^{b}, 0<b<1$. We assume that conditions (A1) and (A2) hold for $X$. We further assume that

$$
\sup _{|t-s|<h}\left|\phi_{k}(s)-\phi_{k}(t)\right| \leq C_{k}|h|^{\zeta} .
$$

If

$$
\sum_{k=1}^{\infty}\left\|\xi_{k}\right\|^{2 a /(a b+1)} C_{k}^{a b /(a b+1)}<\infty
$$

and

$$
\zeta>\frac{1}{a b},
$$

then $\sup _{t \in T}\left|\tilde{X}_{N}(t)\right| \in D_{V, W}$. Moreover,

$$
\begin{aligned}
& \mathrm{P}\left\{\sup _{t \in[0, T]}\left|\tilde{X}_{N}(t)\right|>x\right\} \\
& \leq \frac{1}{x^{a b}}\left(\sum_{k=N+1}^{\infty}\left\|\xi_{k}\right\|^{2 a /(a b+1)} \inf _{t \in[0, T]}\left|\phi_{k}^{a b /(a b+1)}(t)\right|\right. \\
& \\
&+\frac{T^{1 /(a b+1)}\left(\sum_{k=N+1}^{\infty} C_{k}^{a b /(a b+1)}\left\|\xi_{k}\right\|^{2 a /(a b+1)}\right)^{1 /(a b \zeta)}}{2^{a b /(a b+1)} p(1-p)} \frac{a b \zeta\left(\Delta_{N} p\right)^{1-1 /(a b \zeta)}}{a b \zeta-1} \\
& \\
& \\
&\left.+\frac{\Delta_{N}}{1-p}\right),
\end{aligned}
$$

where

$$
\Delta_{N}=\left(\sup _{t, s \in[0, T]}\left(X_{N}(t)-X_{N}(s)\right)\right)^{2 a /(a b+1)}
$$

Proof. Let

$$
\sup _{|t-s|<h}\left|\phi_{k}(t)-\phi_{k}(s)\right| \leq C_{k} \cdot|h|^{\zeta} .
$$


Then

$$
\begin{aligned}
& \sup _{|t-s|<h}\left\|\tilde{X}_{N}(t)-\tilde{X}_{N}(s)\right\|=\sup _{|t-s|<h}\left\|\sum_{k=N+1}^{\infty} \xi_{k}\left(\phi_{k}(t)-\phi_{k}(s)\right)\right\| \\
& \quad \leq\left(\sum_{k=N+1}^{\infty}\left\|\xi_{k}\right\|^{2 a /(a b+1)} \sup _{|t-s|<h} J^{2 a /(a b+1)}\left(\phi_{k}(t)-\phi_{k}(s)\right)\right)^{(a b+1) /(2 a)} \\
& \quad \leq\left(\sum_{k=1}^{\infty}\left\|\xi_{k}\right\|^{2 a /(a b+1)}\left(C_{k}^{b / 2} h^{b \zeta / 2}\right)^{2 a /(a b+1)}\right)^{(a b+1) /(2 a)} \\
& \quad=h^{b \zeta / 2}\left(\sum_{k=1}^{\infty}\left\|\xi_{k}\right\|^{2 a /(a b+1)} C_{k}^{a b /(a b+1)}\right)^{(a b+1) /(2 a)}
\end{aligned}
$$

since $J(z)=z^{b / 2}$ and

$$
\left\|\sum_{k=N+1}^{\infty} \xi_{k}\right\| \leq\left(\sum_{k=N+1}^{\infty}\left\|\xi_{k}\right\|^{2 a /(a b+1)}\right)^{(a b+1) /(2 a)} .
$$

Since $t \in[0, T]$, we have

$$
N(\varepsilon) \leq \frac{T}{2 \delta^{(-1)}(h)}+1,
$$

where $\delta(h)$ can be chosen such that

$$
\delta(h)=h^{b \zeta / 2}\left(\sum_{k=1}^{\infty}\left\|\xi_{k}\right\|^{2 a /(a b+1)} C_{k}^{a b /(a b+1)}\right)^{(a b+1) /(2 a)}
$$

if the series

$$
\sum_{k=N+1}^{\infty}\left\|\xi_{k}\right\|^{2 a /(a b+1)} C_{k}^{a b /(a b+1)}
$$

converges.

By Theorem 2.4

$$
\begin{aligned}
& \mathrm{P}\left\{\sup _{t \in[0, T]}\left|\tilde{X}_{N}(t)\right|>x\right\} \\
& \quad \leq \frac{1}{x^{a b}}\left(\inf _{t \in[0, T]}\left\|\tilde{X}_{N}(t)\right\|^{2 a /(a b+1)}+\frac{1}{p(1-p)} \int_{0}^{\Delta_{N} p}\left(N\left(u^{(a b+1) /(2 a)}\right)\right)^{1 /(a b+1)} d u\right) \\
& \quad \leq \frac{1}{x^{a b}}\left(\inf _{t \in[0, T]}\left\|\tilde{X}_{N}(t)\right\|^{2 a /(a b+1)}\right. \\
& \left.\quad+\frac{1}{p(1-p)} \int_{0}^{\Delta_{N} p}\left(\frac{T}{2 \delta^{(-1)\left(u^{(a b+1) /(2 a)}\right)}+1}\right)^{1 /(a b+1)} d u\right)
\end{aligned}
$$




$$
\begin{aligned}
& \leq \frac{1}{x^{a b}}\left(\inf _{t \in[0, T]}\left\|\tilde{X}_{N}(t)\right\|^{2 a /(a b+1)}\right. \\
& +\frac{1}{p(1-p)} \\
& \left.\times \int_{0}^{\Delta_{N} p}\left(\frac{T\left(\sum_{k=N+1}^{\infty} C_{k}^{a b /(a b+1)}\left\|\xi_{k}\right\|^{2 a /(a b+1)}\right)^{(a b+1) /(a b \zeta)}}{2 u^{(a b+1) /(a b \zeta)}}+1\right)^{1 /(a b+1)} d u\right) \\
& \leq \frac{1}{x^{a b}}\left(\inf _{t \in[0, T]}\left\|\tilde{X}_{N}(t)\right\|^{2 a /(a b+1)}\right. \\
& +\frac{T^{1 / 2 a}\left(\sum_{k=N+1}^{\infty} C_{k}^{a b /(a b+1)}\left\|\xi_{k}\right\|^{2 a /(a b+1)}\right)^{(a b+1) /\left(2 a^{2} b \zeta\right)}}{p(1-p) 2^{a b /(a b+1)}} \\
& \left.\times \int_{0}^{\Delta_{N} p} \frac{d u}{u^{(a b+1) /\left(2 a^{2} b \zeta\right)}}+\frac{\Delta_{0}}{1-p}\right) \\
& \leq \frac{1}{x^{a b}}\left(\sum_{k=N+1}^{\infty}\left\|\xi_{k}\right\|^{2 a /(a b+1)} \inf _{t \in[0, T]}\left|\phi_{k}^{a b /(a b+1)}(t)\right|\right. \\
& +\frac{T^{1 /(a b+1)}\left(\sum_{k=N+1}^{\infty} C_{k}^{a b /(a b+1)}\left\|\xi_{k}\right\|^{2 a /(a b+1)}\right)^{1 /(a b \zeta)}}{p(1-p) 2^{a b /(a b+1)}} \\
& \left.\times \frac{a b \zeta\left(\Delta_{N} p\right)^{1-1 /(a b \zeta)}}{a b \zeta-1}+\frac{\Delta_{N}}{1-p}\right)
\end{aligned}
$$

if the integral

$$
\int_{0}^{\Delta_{N} p} \frac{1}{u^{1 /(a b \zeta)}} d u
$$

is finite, which is the case for

$$
\zeta>\frac{1}{a b}
$$

Corollary 4.1. Assume that

$$
\sup _{|t-s|<h}\left|\phi_{k}(s)-\phi_{k}(t)\right| \leq C_{k}|h|^{\zeta}
$$

A model $X_{N}(t)$ approximates a process $X(t)$ for $t \in[0, T]$ in the space $D_{V, W}(\Omega)$ with given accuracy $æ>0$ and reliability $1-\nu, 0<\nu<1$, which means that

$$
\mathrm{P}\left\{\sup _{t \in[0, T]}\left|\tilde{X}_{N}(t)\right|>\Re\right\} \leq \nu
$$


if

$$
\begin{aligned}
\nu \geq \frac{1}{\mathfrak{x}^{a b}}\left(\sum_{k=N+1}^{\infty}\left\|\xi_{k}\right\|^{2 a /(a b+1)} \inf _{t \in[0, T]}\left|\phi_{k}^{a b /(a b+1)}(t)\right|\right. & \left.+\frac{\Delta_{N}}{1-p}\right), \\
+ & \frac{T^{1 /(a b+1)}\left(\sum_{k=N+1}^{\infty} C_{k}^{a b /(a b+1)}\left\|\xi_{k}\right\|^{2 a /(a b+1)}\right)^{1 /(a b \zeta)}}{2^{a b /(a b+1)} p(1-p)} \frac{a b \zeta\left(\Delta_{N} p\right)^{1-1 /(a b \zeta)}}{a b \zeta-1} \\
\sum_{k=1}^{\infty}\left|C_{k}\right|^{a b /(a b+1)}\left\|\xi_{k}\right\|^{2 a /(a b+1)}<\infty, &
\end{aligned}
$$

and

where

$$
\zeta>\frac{1}{a b}
$$

$$
\Delta_{N}=\left(\sup _{s, t \in[0, T]}\left(X_{N}(t)-X_{N}(s)\right)\right)^{2 a /(a b+1)} .
$$

5. EXAMples OF MODEls OF STOCHASTIC PROCESSES BELONGING TO THE SPACES $D_{V, W}$

In this section we consider stochastic processes $X$ represented in the interval $[0, T]$ in the form of the following series:

$$
X(t)=\sum_{k=1}^{\infty} \xi_{k} \varphi_{k}(t)
$$

where $\xi_{k} \in D_{V, W}$.

Example 5.1. Assume that a process $X(t)$ is represented as follows:

$$
X(t)=\sum_{k=1}^{\infty} \sqrt{2} \xi_{k} \sin (\pi k t)
$$

For this process,

$$
\begin{aligned}
\sup _{|t-s|<h}\left|\phi_{k}(t)-\phi_{k}(s)\right| & =\sup _{|t-s|<h}|\sin (\pi k t)-\sin (\pi k s)| \\
& \leq 2\left|\sin \left(\frac{\pi k h}{2}\right)\right| \leq \pi k h,
\end{aligned}
$$

that is, $C_{k}=\pi k$ and $\zeta=1$ if

$$
a \in\left(\frac{1}{b},+\infty\right)
$$

At the same time,

$$
\sum_{k=N+1}^{\infty}\left\|\xi_{k}\right\|^{2 a /(a b+1)} \inf _{t \in[0, T]}|\sin (\pi k t)|^{a b /(a b+1)}=0
$$


and

$$
\begin{aligned}
& \varepsilon_{N}=\sup _{t, s \in[0, T]}\left\|X_{N}(t)-X_{N}(s)\right\| \\
& \leq \sup _{t, s \in[0, T]}\left(\sum_{k=N}^{\infty}\left\|\sqrt{2} \xi_{k}(\sin (\pi k t)-\sin (\pi k s))\right\|^{2 a /(a b+1)}\right)^{(a b+1) /(2 a)} \\
& \leq 2^{3 b / 4}\left(\sum_{k=N}^{\infty}\left\|\xi_{k}\right\|^{2 a /(a b+1)}\right)^{(a b+1) /(2 a)}, \\
& \Delta_{N}=2^{3 a b /(2 a b+2)} \sum_{k=N}^{\infty}\left\|\xi_{k}\right\|^{2 a /(a b+1)} .
\end{aligned}
$$

The value of $N$ is chosen for the given accuracy $æ$, reliability $1-\nu$, and constant $\theta$. The inequality

$$
\begin{aligned}
\nu \geq \frac{1}{\mathfrak{x}^{a b}} & \left(\frac{a b T^{1 /(a b+1)}\left(\theta^{2 a /(a b+1)} 2^{3 a b /(2 a b+2)} \sum_{k=1}^{\infty}\left\|\xi_{k}\right\|^{2 a /(a b+1)}\right)^{1-1 /(a b)}}{\theta^{2 a /(a b+1)}\left(1-\theta^{2 a /(a b+1)}\right)(a b-1)}\right. \\
& \left.\times\left(\sum_{k=N+1}^{\infty}\left\|\xi_{k}\right\|^{2 a /(a b+1)}(\pi k)^{a b /(a b+1)}\right)^{1 /(a b)}+\frac{2^{3 a b /(2 a b+2)} \sum_{k=1}^{\infty}\left\|\xi_{k}\right\|^{2 a /(a b+1)}}{1-\theta^{2 a /(a b+1)}}\right)
\end{aligned}
$$

can be used to choose $N$ provided that

$$
\sum_{k=1}^{\infty}(\pi k)^{a b /(a b+1)}\left\|\xi_{k}\right\|^{2 a /(a b+1)}<\infty .
$$

The same conditions can be used to study the process

$$
X(t)=\sum_{k=1}^{\infty} \sqrt{2} \xi_{k} \cos (\pi k t) .
$$

Moreover, the same constants can be used for processes (16) as in the case of processes (15).

Example 5.2. Let a stochastic process $X(t)$ be represented in the following form:

$$
X(t)=\sum_{k=0}^{\infty} \xi_{k}\left(A_{k} \sin \left(B_{k} t\right)+C_{k} \cos \left(D_{k} t\right)\right),
$$

where $A_{k}>0$ and $C_{k}>0$. In this case,

$$
\begin{aligned}
& \sup _{|t-s|<h}\left|\left(A_{k} \sin \left(B_{k} t\right)+C_{k} \cos \left(D_{k} t\right)\right)-\left(A_{k} \sin \left(B_{k} s\right)+C_{k} \cos \left(D_{k} s\right)\right)\right| \\
& \quad=\sup _{|t-s|<h}\left|2 A_{k} \sin \left(B_{k} \frac{t-s}{2}\right) \sin \left(B_{k} \frac{t+s}{2}\right)-2 C_{k} \sin \left(D_{k} \frac{t-s}{2}\right) \sin \left(D_{k} \frac{t+s}{2}\right)\right| .
\end{aligned}
$$

Since $\sin x \leq x^{\alpha}, 0<\alpha \leq 1$,

$$
\begin{aligned}
& \sup _{|t-s|<h}\left|2 A_{k} \sin \left(B_{k} \frac{t-s}{2}\right) \sin \left(B_{k} \frac{t+s}{2}\right)-2 C_{k} \sin \left(D_{k} \frac{t-s}{2}\right) \sin \left(D_{k} \frac{t+s}{2}\right)\right| \\
& \quad \leq 2 A_{k}\left|\sin \left(B_{k} h / 2\right)\right|+2 C_{k}\left|\sin \left(D_{k} h / 2\right)\right| \leq 2^{1-\alpha}\left(A_{k} B_{k}^{\alpha}+C_{k} D_{k}^{\alpha}\right) h^{\alpha} .
\end{aligned}
$$

The corresponding integral converges if

$$
a \in\left(\frac{1}{\alpha b},+\infty\right) .
$$


Put $E_{k}:=2^{1-\alpha}\left|A_{k} B_{k}^{\alpha}+C_{k} D_{k}^{\alpha}\right|$. In this case, $\inf _{t \in[0, T]}\left\|\tilde{X}_{N}\right\|$ and $\Delta_{0}$ do not depend on the coefficients and cannot be evaluated explicitly. Thus we first choose the reliability $1-\nu$, accuracy $æ$, and the constant $\theta$. Then we use the inequality

$$
\begin{aligned}
\nu \geq \frac{1}{\mathfrak{x}^{a b}}\left(\inf _{t \in[0, T]}\left\|\tilde{X}_{N}(t)\right\|^{2 a /(a b+1)}\right. & \\
& +\frac{\alpha a b T^{1 /(a b+1)}\left(\sum_{k=N+1}^{\infty}\left\|\xi_{k}\right\|^{2 a /(a b+1)} E_{k}^{a b /(a b+1)}\right)^{1 /(a b \alpha)}}{\theta^{2 a /(a b+1)}\left(1-\theta^{2 a /(a b+1)}\right)} \\
& \left.\times \frac{\left(\Delta_{N} p\right)^{1-1 /(a b \alpha)}}{a b \alpha-1}+\frac{\Delta_{N} p}{1-\theta^{2 a /(a b+1)}}\right)
\end{aligned}
$$

to evaluate the number $N$ under the assumption that

$$
\sum_{k=1}^{\infty} E_{k}^{a b /(a b+1)}\left\|\xi_{k}\right\|^{2 a /(a b+1)}<\infty .
$$

\section{BIBLIOGRAPHY}

1. Yu. V. Kozachenko and O. M. Moklyachuk, Stochastic processes in the spaces $D_{V, W}$, Teor. Imovir. Mat. Stat. 82 (2010), 56-66; English transl. in Theor. Probability and Math. Statist. 82 (2011), 43-56. MR2790483 (2011m:60101)

2. V. V. Buldygin and Yu. V. Kozachenko, Metric Characterization of Random Variables and Random Processes, TViMS, Kiev, 1998; English transl., American Mathematical Society, Providence, Rhode Island, 2000. MR1743716 (2001g:60089)

3. Yu. V. Kozachenko, On the distribution of the supremum of random processes in quasi-Banach $K_{\sigma}$-spaces, Ukrain. Mat. Zh. 51 (1999), no. 7, 918-930; English transl. in Ukrainian Math. J. 51 (2000), no. 7, 1029-1043. MR.1727696 (2000k:60066)

4. V. V. Buldygin, Convergence of Random Elements in Topological Spaces, Naukova Dumka, Kiev, 1980. (Russian) MR734899 (84m:60011)

5. E. A. Abzhanov and Yu. V. Kozachenko, Some properties of random processes in Banach $K_{\sigma}{ }^{-}$ spaces, Ukrain. Mat. Zh. 37 (1985), no. 3, 275-280; English transl. in Ukr. Math. J. 37 (1986), no. 3, 209-213. MR795565 (87m:60095)

6. E. A. Abzhanov and Yu. V. Kozachenko, Random processes in $K_{\sigma}$-spaces of random variables, Probabilistic Methods for the Investigation of Systems with an Infinite Number of Degrees of Freedom (A. V. Skorokhod, ed.), Institute of Mathematics, Academy of Science of Ukrain. SSR, Kiev, 1986, pp. 4-11, Russian. MR895373 (88g:60101)

7. Yu. V. Kozachenko, Random processes in Orlicz spaces. I, Teor. Veroyatnost. i Mat. Statist. 30 (1984), 92-107; English transl. in Theory Probab. Math. Statist. 30 (1985), 103-117. MR800835 $(86 \mathrm{~m}: 60111)$

8. Yu. V. Kozachenko, Random processes in Orlicz spaces. II, Teor. Veroyatnost. i Mat. Statist. 31 (1984), 44-50; English transl. in Theory Probab. Math. Statist. 31 (1985), 51-58. MR816125 (87b:60063)

Department of Probability Theory, Statistics, and Actuarial Mathematics, Faculty for Mechanics and Mathematics, National Taras Shevchenko University, Academician Glushkov Avenue 2, Kiev 03127, Ukraine

E-mail address: yvk@univ.kiev.ua

Department of Probability Theory, Statistics, and Actuarial Mathematics, Faculty for Mechanics and Mathematics, National Taras Shevchenko University, Academician Glushkov Avenue 2, Kiev 03127, Ukraine

E-mail address: omoklyachuk@ukr.net 